\title{
Does linked selection explain the narrow range of genetic diversity across species?
}

\author{
Graham Coop \\ Center for Population Biology, University of California, Davis. \\ Department of Evolution and Ecology, University of California, Davis \\ gmcoop@ucdavis.edu
}

March 6, 2016

Two observations have puzzled population geneticists since the inception of molecular population genetics. The first is the relatively high level of genetic variation observed in most obligately sexual species. The neutral theory of molecular evolution was developed in part to explain the high levels of diversity. Under a simple neutral model, with constant population size, we should expect the amount of neutral genetic diversity to scale with the product of the population size and mutation rate. Specifically, in a randomly mating diploid population of size $N_{c}$ individuals, at sites that experience a neutral per generation mutation rate of $\mu$, we should expect pairwise diversity $\pi \approx 4 N_{c} \mu$. The second observation, however, is the relatively narrow range of polymorphism across species with vastly different census sizes (see Leffler et al., 2012, for a recent review). As highlighted by Lewontin (1974) in his discussion of the paradox of variation, this seemingly contradicts the prediction of the neutral theory that genetic diversity should scale with the census population size. In eukaryotes, per generation mutation rates appear to vary over just two orders of magnitude $\left(10^{-10}-10^{-8}\right)$, while census population sizes range over many orders of magnitude, from populations consisting of just a few thousand individuals to populations measured in billions of individuals. Therefore, diversity levels should vary over many orders of magnitude, but levels of synonymous genetic diversity in obligately sexual populations differ by only a few, from $0.01-10 \%$ (LEFFLER et al., 2012).

There are a number of explanations for the discrepancy between genetic diversity levels and census population sizes. The first is that the effective size of the population $\left(N_{e}\right)$ is often much lower than the census size, due to high variance in reproductive success and frequent bottlenecks (see CHARLESWORTH, 2009, for a review). The second major explanation, put forward by MAYNARD SMith and HAIGH (1974), is that neutral levels of diversity are also systematically reduced by the effects of linked selection. The sweep of a beneficial allele to fixation removes neutral diversity at linked sites (the hitchhiking effect; Maynard Smith and Haigh, 1974; Kaplan et al., 1989; StePhan et al., 1992) as does the removal of deleterious alleles from the population (background selection; CHARLESWORTH et al., 1995; HUdSON and KAPLAN, 1995b,a; NorDBORG et al., 1996). In fact, a wide range of models of selection predict the removal of neutral diversity linked to selected sites. This is because heritable variance in fitness leads the effect of high variance in reproductive success on levels of diversity to be compounded over generations (Robertson, 1961; Santiago and Caballero, 1995, 1998; Barton, 2000; Gillespie, 1994, 1997; NeHer et al., 2013; Good et al., 2014).

Evidence for the action of linked selection in reducing levels of polymorphism includes a positive correlation between putatively neutral diversity and recombination in a number of species, as, all else being equal, linked selection should be less efficient in removing diversity in regions of high recombination (Aguadé et al., 1989; Begun and Aquadro, 1992; Wiehe and Stephan, 1993; Cutter and Choi, 2010; Hellmann et al., 2008; Cai et al., 2009; Cutter and Payseur, 2013). In addition, levels of putatively neutral diversity are often lower in regions with a higher density of functional sites, where we expect more selected alleles to arise (Andolfatto, 2007; MACPHERson et al., 2007; MCVicker et al., 2009; Sattath et al., 2011; Hernandez et al., 2011; Beissinger et al., 2015). In large populations, selective sweeps and other forms of linked selection may come to dominate genetic drift as a source of stochasticity in allele frequencies, establishing an upper limit to levels of diversity (KAPLAN et al., 1989; Gillespie, 2000). 


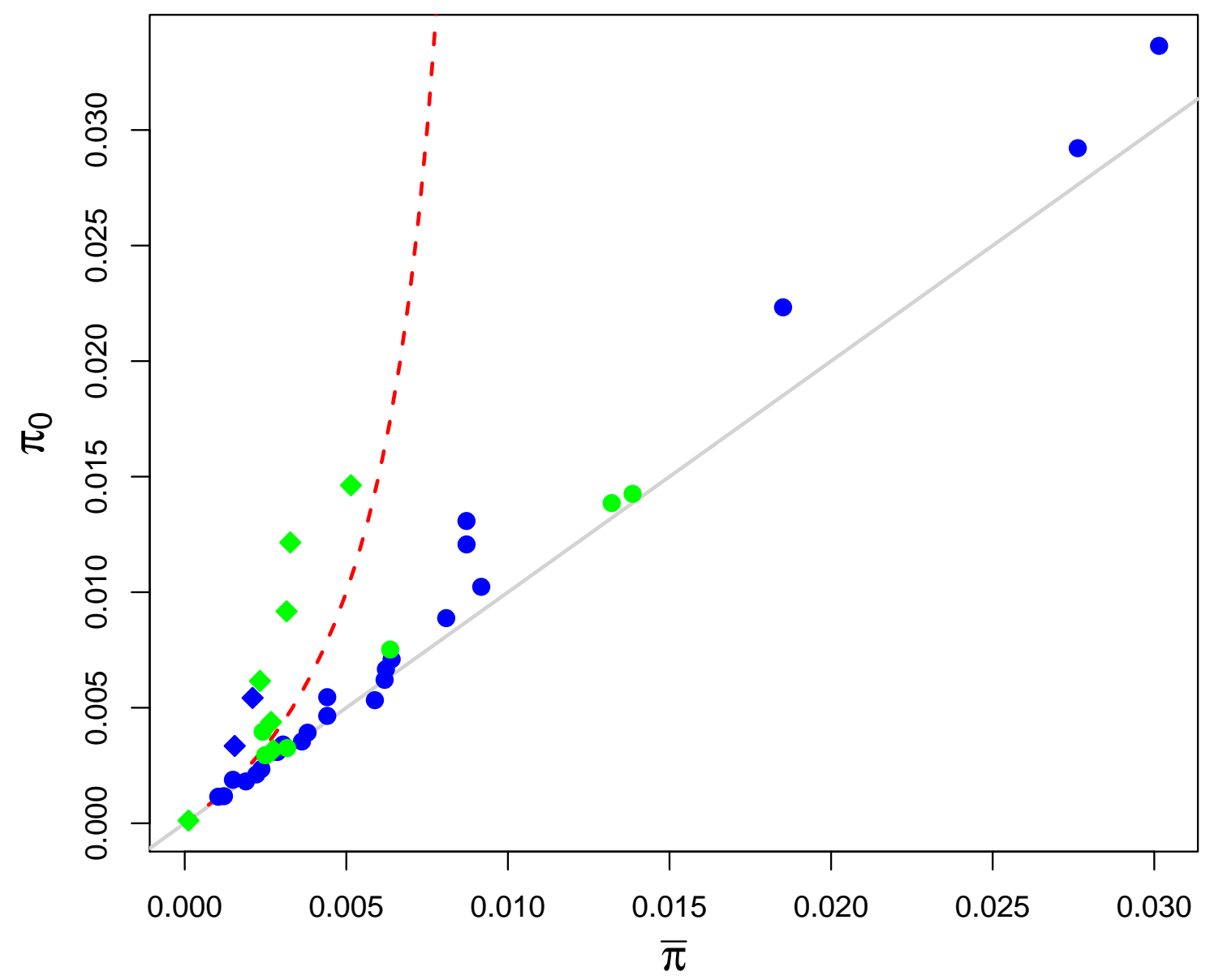

Figure 1: The genome-wide average of four-fold degenerate site diversity $\left(\pi_{a v g}\right)$ plotted against Corbett-Detig et al. (2015)'s estimates of the level of genetic diversity in the absence of linked selection $\left(\pi_{0}\right)$ across species. Animal species are shown in blue, plant species are in green. Species with high selfing rates, as identified by CoRBetT-Detig et al. (2015), are shown as diamonds. The grey line shows the $x=y$ line that we would expect in the absence of linked selection. The red line shows the type of relationship that we would expect to see if linked selection were a general explanation for the narrow range of genetic diversity. This example relationship was obtained by using a range of $N$ in eqn. (1), setting $\mu_{B P}=10^{-9}, r=10^{-8}$, and $\nu_{B P}=10^{-11}$ with $J=10^{-4}$ (roughly corresponding to a sweep of $\left.s=10^{-4}\right)$. I ignore background selection in this prediction for the sake of simplicity. 
bioRxiv preprint doi: https://doi.org/10.1101/042598; this version posted March 7, 2016. The copyright holder for this preprint (which was

To understand the contributions of the two explanations to levels of diversity, it is helpful to distinguish between the average observed level of neutral polymorphism in the genome $(\bar{\pi})$ and that expected in the absence of linked selection $\left(\pi_{0}\right)$. Our idealized neutral level of variation in which $\pi_{0} \approx 4 N_{e} \mu$, reflects the effective size of the population $\left(N_{e}\right)$ in the absence of linked selection ( here $N_{e}$ is not estimated simply from putatively neutral diversity levels genome-wide). To illustrate this point, take the extreme scenario in which linked selection explains nearly all of the deficit in variation in species with large census sizes, with fluctuations in population size playing a minor role. In these species, $\bar{\pi}$ should be orders of magnitude smaller than $\pi_{0}$, and $N_{e}$ should be roughly the same order of magnitude as the census size. In contrast, if fluctuations in population size explain most of the deficit, then $\bar{\pi}$ should be close to $\pi_{0}$ for all species, while $N_{e}$ would be many orders of magnitude lower than census population sizes for species with large population sizes.

Recently, Corbetr-Detig et al. (2015) sought to test the idea that linked selection explains the narrow range of genetic diversity. They compiled published genomic data and genetic maps from 40 obligately sexual species to obtain an impressive dataset for comparative populations genomics. In these data, they found a positive correlation between diversity at four-fold degenerate sites and recombination rates in many species. To estimate the impact of linked selection, they then fit a simple model of hitchiking and background selection to the relationship between recombination rate per base $(r)$ and $\pi$ in genomic windows within each species (also factoring in variation in gene density). Specifically, they fit a model where

$$
\mathbb{E}[\pi]=\frac{\pi_{0} B(r) r}{2 N_{e} B(r) J \nu+r}
$$

where $\pi_{0}=4 N_{e} \mu$ is the expected diversity in the absence of linked selection, $1 / B(r)$ is the increase in the rate of coalescence (genetic drift) due to background selection, $\nu$ is the rate of sweeps per base pair, and $J$ is related to the probability that a particular sweep forces a pair of lineages to coalesce averaged over the distance to the sweep (see COOP and RALPH, 2012, for more discussion). For full sweeps of an allele with an additive selection coefficient $s, J \approx \log \left(2 N_{e} s\right) / s$.

CorbetT-Detig et al. (2015) found that their estimated reduction in diversity due to linked selection $\left(1-\bar{\pi} / \pi_{0}\right)$ is positively correlated with measures of range size and inversely correlated with body size across species, both of which are correlates of the census population size $\left(N_{c}\right)$. The authors interpreted these findings as evidence that "pervasive natural selection constrains neutral diversity and provides an explanation for why neutral diversity does not scale as expected with population size".

Stronger linked selection in species with larger population sizes is indeed qualitatively consistent with MAYNARD SMITH and HAIGH (1974)'s explanation of the narrow range of diversity (KAPLAN et al., 1989). But the hypothesis that linked selection is the major contributor to the narrow range of diversity is a quantitative prediction and requires linked selection to dominate genetic drift by orders of magnitude in species with large population sizes (KAPLAN et al., 1989; GILlESPIE, 2000). I present a reanalysis of the results of CORBETT-Detig et al. (2015), focusing on these quantitative predictions.

Across the 40 species studied, the average level of diversity $\bar{\pi}$ ranges from $0.1 \%-3.0 \%$ in animals and $0.012 \%-1.5 \%$ in plants. The authors' predictions of the range of diversity in the absence of linked selection $\left(\pi_{0}\right)$ are surprisingly similar (from $0.12 \%-3.36 \%$ in animals, and $0.012 \%-1.5 \%$ in plants). Even the maximum estimated reduction in genome-wide diversity $(70 \%)$ is less than one order of magnitude. In order for linked selection to explain the paradox of variation, however, the estimated reductions would need to vary over several orders of magnitude to explain the reduction in variation in species over the full range of census population sizes.

To further illustrate this point, I plot the observed genome-wide average $(\bar{\pi})$ for each species versus the estimated level of diversity in the absence of linked selection $\left(\pi_{0}\right)$ (Figure 1). If linked selection strongly constrained levels of diversity, we should expect to see a pattern somewhat like the red line, in which species with very high levels of diversity in the absence of linked selection $\left(\pi_{0}\right)$ only have moderately higher levels of genome-wide average diversity $(\bar{\pi})$. Instead, predicted diversities fall mostly along the $x=y$ line - the result that we would expect if linked selection had only a limited impact on levels of diversity in large populations and diversity levels scaled with effective population sizes estimated in the absence of linked selection. This finding is consistent with the idea that demographic fluctuations are the principal determinant of levels of diversity among species. Interestingly, selfing species (shown in italics; Figure 1) seem to show the best evidence of large reductions in diversity due to linked selection, perhaps due to their much reduced effective rate of recombination (NoRDBORG, 1997; CHARLESWORTH et al., 1997; Charlesworth and Wright, 2001). 
bioRxiv preprint doi: https://doi.org/10.1101/042598; this version posted March 7, 2016. The copyright holder for this preprint (which was

These results seemingly do not support the idea that linked selection dominates drift in the removal of diversity from large populations. That said, caution is warranted, as $\pi_{0}$ was estimated by fitting a simple model of linked selection. In particular, these estimates assume that in regions of high recombination (and low enough gene density), we can hope to see close to $\pi_{0}$, i.e. that the curve of $\pi$ against $r$ plateaus to $\pi_{0}$ as drift comes to dominate linked selection. However, if linked selection truly dominates genetic drift, we may have little indication as to the true effective size of the population (i.e. in the absence of linked selection). For example, if the rate of coalescence due to hitchhiking, on the time-scale of genetic drift, is much higher than the highest recombination rate per base pair in the genome $\left(2 N_{e} \nu J \gg r\right)$, then

$$
\mathbb{E}(\pi) \approx \frac{2 r \mu}{J \nu_{B P}}
$$

i.e. diversity increases linearly with recombination rate, and is completely independent of the rate of drift (Kaplan et al., 1989; Gillespie, 2000; CoOP and RalPh, 2012).

To begin to explore whether levels of diversity in high recombination regions are still subject to strong linked selection or represent a balance of drift and mutation, in Figure 2 I plot the slope and correlation of pairwise diversity regressed against recombination both genome-wide and in windows of high recombination, using the binned data of CORBETT-DETIG et al. (2015). If linked selection dominated drift, we would expect to still see a positive slope between diversity and recombination even in high recombination regions. However, we fail to observe this pattern (right column of Figure 2) in the majority of species. The restricted recombination range in the second column means that we should expect the correlations to potential be lower, and the slope estimates to be noiser (i.e. have larger confidence intervals). However, the low upper confidence intervals for the slope in the top quartile of recombination regions suggest that the relationship between diversity and recombination is plateauing in most species. Thus there is preliminary evidence that recombination - and thus presumably linked selection - is not a strong determinant of levels of diversity in high recombination regions.

These findings suggest that, in many species, levels of diversity in high recombination regions potentially do offer good estimates of the effective population size in the absence of linked selection. Thus, even in the apparent absence of linked selection, the range of effective population sizes appears tightly constrained across species that differ vastly in their census sizes.

This is not to say that linked selection is not an important factor in understanding levels of diversity within genomes. Undoubtedly as genetic maps, functional annotations, and statistical methodologies improve, our appreciation of the effects of linked selection will increase as well (MCVICKER et al., 2009; Comeron, 2014; Elyashiv et al., 2014). For example, Elyashiv et al. (2014) inferred an average diversity reduction of only $34-36 \%$ in Drosophila melanogaster using just the relationship between recombination rates and diversity, similar to that used here, and found no effect of linked selection in the upper $1 \%$ quantile of predicted diversity levels. However, by fitting a more detailed model that incorporated a range of annotations and variation in the strength of selection, ELYASHIV et al. (2014) estimated an average $77-89 \%$ reduction in diversity due to selection on linked sites, and concluded that no genomic window was entirely free of the effect of selection. Nonetheless, it seems unlikely that our estimates of the effect of linked selection (based on current models) will approach the orders of magnitude needed for linked selection to be the major explanation of the paradox of variation.

In summary, while we still lack a full picture, a reanalysis of the results of CoRBETT-DetiG et al. (2015) does not support the idea that linked selection is the major factor in shaping diversity levels among obligate sexual species. As highlighted by CORBETT-DETIG et al. (2015), linked selection does play a major role in shaping variation levels along many species' genomes, sometimes contributing more to variation in allele frequencies than genetic drift alone. As such, CoRBETT-Detig et al. (2015)'s results reinforce the view that linked selection must be more thoroughly incorporated into our null models for population genetics. But it may well be the case that the major driver of variation in levels of diversity among sexual species, and therefore the major cause of the discrepancy between levels of diversity and population size, is fluctuations in population size. 

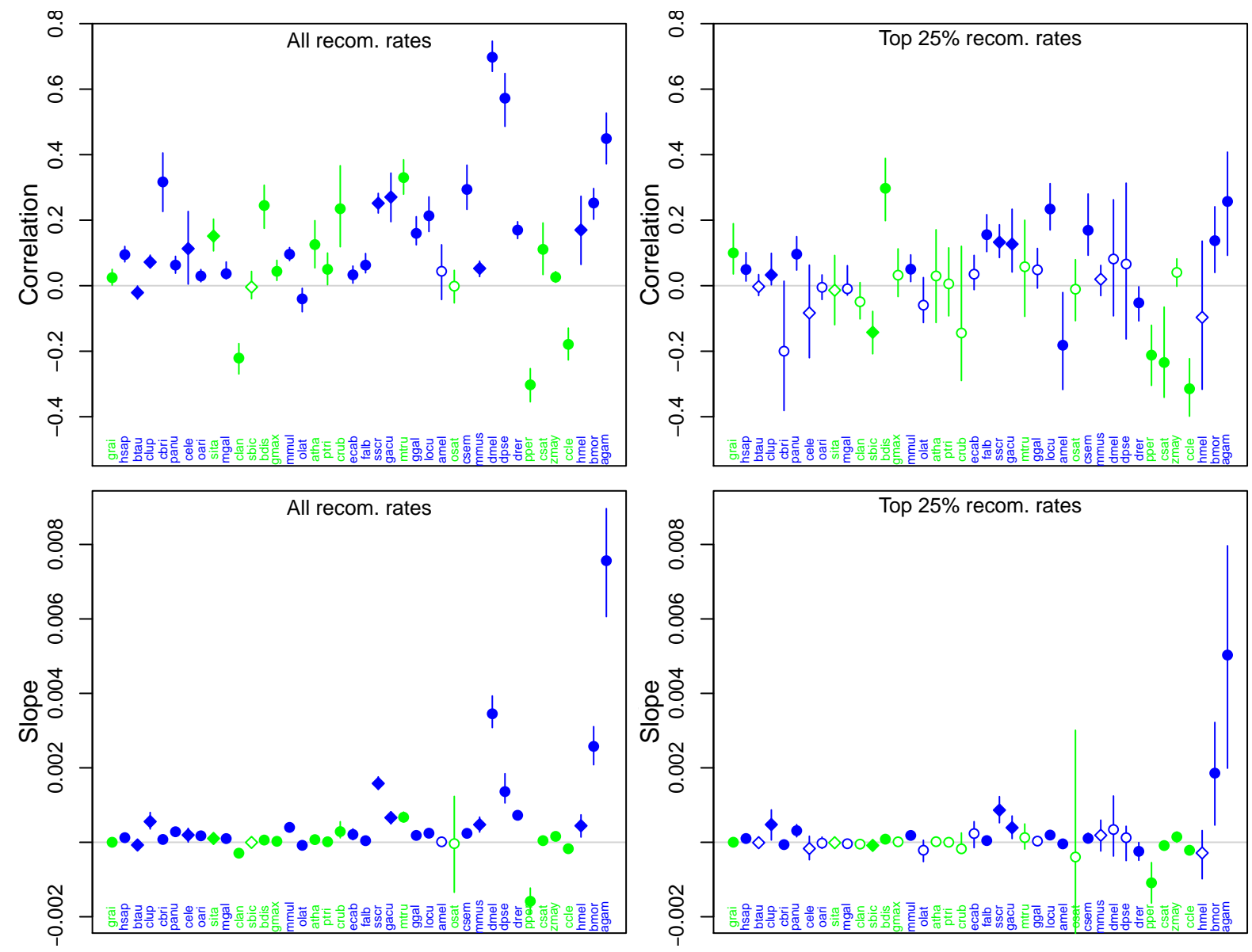

Figure 2: Top row the Pearson correlation coefficient between recombination rate and genetic diversity at fourfold degenerate sites in $500 \mathrm{~kb}$ windows, colored and labeled by species. On the left, all windows are used, on the right only windows with recombination rates in the top quartile. Species are arranged left to right in order of increasing average levels of diversity. Points are colored as in Figure 1. The line shows the $95 \%$ bootstrap confidence interval for the correlation coefficient calculated by sampling $500 \mathrm{~kb}$ windows with replacement. Open points indicate that the $95 \%$ confidence interval overlaps zero. Bottom row the slopes of the linear regression of diversity on recombination rate (with the same layout as the top panel). 


\section{Acknowledgements}

I thank Jeremy Berg, Gideon Bradburd, Vince Buffalo, Nancy Chen, Chuck Langley, Molly Przeworski, Jeff Ross-Ibarra, and Guy Sella for useful discussions based on earlier drafts of this note. I thank Russ Corbett-Detig and Tim Sackton for their feedback on an earlier draft and for making their code and data publicly available.

\section{References}

Aguadé, M., N. Miyashita, and C. H. Langley, 1989 Reduced variation in the yellow-achaete-scute region in natural populations of Drosophila melanogaster. Genetics 122: 607-615.

Andolfatto, P., 2007 Hitchhiking effects of recurrent beneficial amino acid substitutions in the Drosophila melanogaster genome. Genome Res. 17: 1755-1762.

Barton, N. H., 2000 Genetic hitchhiking. Philos. Trans. R. Soc. Lond., B, Biol. Sci. 355: 1553-1562.

Begun, D. J. and C. F. AquADro, 1992 Levels of naturally occurring DNA polymorphism correlate with recombination rates in D. melanogaster. Nature 356: 519-520.

Beissinger, T. M., L. Wang, K. Crosby, A. Durvasula, M. B. Hufford, and J. Ross-Ibarra, 2015 Recent demography drives changes in linked selection across the maize genome. bioRxiv: 031666.

Cai, J. J., J. M. Macpherson, G. Sella, and D. A. Petrov, 2009 Pervasive hitchhiking at coding and regulatory sites in humans. PLoS Genet. 5: e1000336.

Charlesworth, B., 2009 Fundamental concepts in genetics: effective population size and patterns of molecular evolution and variation. Nat. Rev. Genet. 10: 195-205.

Charlesworth, B., M. Nordborg, and D. Charlesworth, 1997 The effects of local selection, balanced polymorphism and background selection on equilibrium patterns of genetic diversity in subdivided populations. Genet. Res. 70: 155-174.

Charlesworth, D., B. Charlesworth, and M. T. Morgan, 1995 The pattern of neutral molecular variation under the background selection model. Genetics 141: 1619-1632.

Charlesworth, D. and S. I. Wright, 2001 Breeding systems and genome evolution. Current Opinion in Genetics \& Development 11(6): 685 - 690 .

Comeron, J. M., 2014 Background selection as baseline for nucleotide variation across the Drosophila genome. PLoS Genet 10(6): e1004434.

Coop, G. and P. RAlPh, 2012, Sep)Patterns of neutral diversity under general models of selective sweeps. Genetics 192(1): 205-224.

Corbett-Detig, R. B., D. L. Hartl, and T. B. Sackton, 2015, Apr)Natural selection constrains neutral diversity across a wide range of species. PLoS Biol. 13(4): e1002112.

Cutter, A. D. and J. Y. Choi, 2010 Natural selection shapes nucleotide polymorphism across the genome of the nematode Caenorhabditis briggsae. Genome Res. 20: 1103-1111.

Cutter, A. D. and B. A. Payseur, 2013 Genomic signatures of selection at linked sites: unifying the disparity among species. Nature Reviews Genetics 14(4): 262-274.

Elyashiv, E., S. Sattath, T. T. Hu, A. Strustovsky, G. McVicker, P. Andolfatto, G. Coop, and G. SElla, 2014 A genomic map of the effects of linked selection in Drosophila. arXiv preprint arXiv:1408.5461.

Gillespie, J., 1994 Alternatives to the Neutral Theory. In B. Golding (Ed.), Non-Neutral Evolution. Theories and Molecular Data. Chapman and Hall. 
bioRxiv preprint doi: https://doi.org/10.1101/042598; this version posted March 7,2016. The copyright holder for this preprint (which was

Gillespie, J. H., 1997 Junk ain’t what junk does: neutral alleles in a selected context. Gene 205: 291-299.

Gillespie, J. H., 2000 Genetic drift in an infinite population. The pseudohitchhiking model. Genetics 155: 909-919.

Good, B. H., A. M. Walczak, R. A. Neher, and M. M. Desai, 2014, Mar)Genetic diversity in the interference selection limit. PLoS Genet. 10(3): e1004222.

Hellmann, I., Y. Mang, Z. Gu, F. Li, P. de la Vega, A. Clark, and R. Nielsen, 2008 Population genetic analysis of shotgun assemblies of genomic sequences from multiple individuals. Genome Research 18: 1020-1029.

Hernandez, R. D., J. L. Kelley, E. Elyashiv, S. C. Melton, A. Auton, G. McVean, G. Sella, and M. Przeworski, 2011 Classic selective sweeps were rare in recent human evolution. Science 331: 920-924.

Hudson, R. R. and N. L. Kaplan, 1995a Deleterious background selection with recombination. Genetics 141: 1605-1617.

Hudson, R. R. and N. L. Kaplan, 1995b The coalescent process and background selection. Philos. Trans. R. Soc. Lond., B, Biol. Sci. 349: 19-23.

Kaplan, N. L., R. R. Hudson, and C. H. Langley, 1989 The hitchhiking effect revisited. Genetics 123: 887-899.

Leffler, E. M., K. Bullaughey, D. R. Matute, W. K. Meyer, L. Segurel, A. Venkat, P. AnDolfatto, and M. Przeworski, 2012 Revisiting an old riddle: what determines genetic diversity levels within species? PLoS Biol. 10(9): e1001388.

Lewontin, R. C., 1974 The Genetic Basis of Evolutionary Change. Columbia University Press, New York.

Macpherson, J. M., G. Sella, J. C. Davis, and D. A. Petrov, 2007 Genomewide spatial correspondence between nonsynonymous divergence and neutral polymorphism reveals extensive adaptation in Drosophila. Genetics 177: 2083-2099.

Maynard Smith, J. and J. Haigh, 1974 The hitch-hiking effect of a favourable gene. Genet. Res. 23: 23-35.

McVicker, G., D. Gordon, C. Davis, and P. Green, 2009 Widespread genomic signatures of natural selection in hominid evolution. PLoS Genet. 5: e1000471.

Neher, R. A., T. A. Kessinger, and B. I. Shraiman, 2013, Sep)Coalescence and genetic diversity in sexual populations under selection. Proc. Natl. Acad. Sci. U.S.A. 110(39): 15836-15841.

Nordborg, M., 1997 Structured coalescent processes on different time scales. Genetics 146: 1501-1514.

Nordborg, M., B. Charlesworth, and D. Charlesworth, 1996 The effect of recombination on background selection. Genet. Res. 67: 159-174.

Robertson, A., 1961 Inbreeding in artificial selection programmes. Genet. Res. 2: 189-194.

Santiago, E. and A. Caballero, 1995 Effective size of populations under selection. Genetics 139: 1013-1030.

Santiago, E. and A. Caballero, 1998 Effective size and polymorphism of linked neutral loci in populations under directional selection. Genetics 149: 2105-2117.

Sattath, S., E. Elyashiv, O. Kolodny, Y. Rinott, and G. Sella, 2011 Pervasive adaptive protein evolution apparent in diversity patterns around amino acid substitutions in Drosophila simulans. PLoS Genet. 7: e1001302. 
Stephan, W., T. Wiehe, and M. Lenz, 1992 The effect of strongly selected substitutions on neutral polymorphism: analytical results based on diffusion theory. Theor. Popul. Biol. 41: 237-254.

Wiene, T. H. and W. Stephan, 1993 Analysis of a genetic hitchhiking model, and its application to DNA polymorphism data from Drosophila melanogaster. Mol. Biol. Evol. 10: 842-854. 